\title{
Wind tunnel measurements of pollutant turbulent fluxes in urban intersections
}

\author{
Matteo Carpentieri ${ }^{*}$, Paul Hayden, Alan G. Robins \\ EnFlo, FEPS, University of Surrey \\ Published in: Atmospheric Environment 46, 669-674 (2012) \\ DOI: 10.1016/j.atmosenv.2011.09.083
}

\begin{abstract}
Wind tunnel experiments have been carried out at the EnFlo laboratory to measure mean and turbulent tracer fluxes in geometries of real street canyon intersections. The work was part of the major DAPPLE project, focussing on the area surrounding the intersection between Marylebone Road and Gloucester Place in Central London, UK. Understanding flow and dispersion in urban streets is a very important issue for air quality management and planning, and turbulent mass exchange processes are important phenomena that are very often neglected in urban modelling studies. The adopted methodology involved the combined use of laser Doppler anemometry and tracer concentration measurements. This methodology was applied to quantify the mean and turbulent flow and dispersion fields within several street canyon intersections. Vertical profiles of turbulent tracer flux were also measured. The technique, despite a number of limitations, proved reliable and allowed tracer balance calculations to be undertaken in the selected street canyon intersections. The experience gained in this work will enable much more precise studies in the future as issues affecting the accuracy of the experimental technique have been identified and resolved.
\end{abstract}

Keywords: Urban canopy, Concentration measurements, Pollutant exchanges, Laser Doppler anemometry, Vertical exchanges

\section{Introduction}

High pollution levels are often associated with street canyons, that is in urban streets flanked by buildings on both sides. Street canyons are not infinitely long or isolated entities in cities, but they are often segmented and connected to other canyons at urban intersections. There have been only a few dispersion studies focussing on urban canyon intersections, where flow patterns and dispersion behaviour can be markedly different to those in idealised, isolated street canyons (Hoydysh and Dabberdt, 1994; Carpentieri et al., 2009; Carpentieri and Robins, 2010).

Urban canyon intersections constitute pollutant exchange areas between several streets, and between the street canopy and the flow above. Understanding these exchange processes is therefore important in order to develop local and neighbourhood scale dispersion models for urban areas. This is not easy as the geometry of real intersections leads to complex threedimensional flows and associated dispersion conditions. In addition to mean flow processes, turbulent fluxes may play a significant role in exchanging pollutants between streets and with the flow above the canopy (Soulhac, 2000; Caton et al., 2003). Despite this, most street canyon and street intersection dispersion modelling studies focus only on mean fluxes, due to the difficulties arising in measuring concentration and velocity fluctuations at the same time and location.

Conventional measurement techniques have been applied in the past to measure turbulent mass fluxes (see, e.g., Fackrell and Robins, 1982; Zhu et al., 1988; Lemoine et al., 1997), but never used to measure fluxes in street canyons. Dezső-Weidinger et al. (2003) developed an

\footnotetext{
** Corresponding author - University of Surrey, Civil Engineering (C5), Guildford GU2 7XH, UK, Tel +44 (0)1483 686657, m.carpentieri@surrey.ac.uk
} 
alternative measuring technique involving Particle Image Velocimetry (PIV) and Particle Tracking Velocimetry (PTV) and applied it to a two-dimensional street canyon with a regular shape in a wind tunnel. They were particularly interested in assessing the proportionality between turbulent mass fluxes and concentration gradients, finding proportionality in the 2D shear region, but not within the canyon. However, they did not try to calculate integral mass fluxes through the interface between the canopy and the region above. Caton et al. (2003) applied a similar PTV technique in a water flume in order to validate their model for pollutant mass exchange between the canopy and the region above. Only mean mass fluxes were estimated, though, while no attempts were made to measure turbulent fluxes.

An interesting technique for measuring scalar fluxes in wind tunnel models of street canyons and, more generally, urban areas was developed by Barlow and Belcher (2002) using naphthalene sublimation (see also Barlow et al., 2004; Pascheke et al., 2008). This experimental technique can be used to estimate space averaged total mass fluxes, thus including turbulent fluxes, but cannot distinguish between the mean and turbulent components. A similar technique was used by Narita (2007), with water evaporation instead of naphthalene.

The research presented in this paper addresses the issue of measuring turbulent fluxes in urban areas. An experimental technique for directly measuring both mean and turbulent tracer fluxes in small scale models of urban areas has been developed. It involves the combined use of a two-component laser Doppler anemometer (LDA) and a fast response flame ionisation detector (FFID). The two instruments had already been used separately in order to estimate the mean pollutant fluxes in and around a street canyon intersection (Carpentieri et al., 2009; Carpentieri and Robins, 2010) - they were used together in the present work in order to be able to separately measure the turbulent tracer fluxes as well. Once developed, the technique was then applied to a small-scale model of a real urban area, in central London, UK.

The need to develop a technique to measure turbulent mass fluxes arose from the outcomes of previous experimental studies (Carpentieri et al., 2009; Carpentieri and Robins, 2010). The work was part of part of DAPPLE (Dispersion of Air Pollution and its Penetration into the Local Environment), a 4-year UK Engineering and Physical Sciences Research Council (EPSRC) funded project within the Engineering for Health, Infrastructure and Environment Programme (Arnold et al., 2004) that continued under a 3-year follow-up project (DAPPLE-HO) funded by the UK Home Office (Wood et al., 2009).

The DAPPLE site (e.g. see also Carpentieri et al., 2009) is located around the intersection between Marylebone Road and Gloucester Place in Central London, UK. The roads intersect perpendicularly and Marylebone Road runs approximately from west-south-west to east-northeast. The average building height is approximately $22 \mathrm{~m}$. As it is a real site, the heights and sizes of the buildings and streets are all different and this strongly influences the pollutant dispersion mechanism within the intersection (Carpentieri et al., 2009; Carpentieri and Robins, 2010).

\section{Experimental set-up and strategy}

The experiments were carried out in the boundary layer wind tunnel of the Environmental Flow Research Centre (EnFlo), at the University of Surrey, UK. This is an open circuit wind tunnel with a $20 \mathrm{~m}$ long, $3.5 \mathrm{~m}$ wide and $1.5 \mathrm{~m}$ high working section. The wind speed can be in the range 0.3 to $3.5 \mathrm{~m} \mathrm{~s}^{-1}$, and the facility is capable of simulating both stable and unstable atmospheric conditions, although this feature was not used in this study. Reference flow conditions are measured by two ultrasonic anemometers, one held at a fixed location and the other positioned as required, and two propeller anemometers mounted on either side of the traverse carriage; the motor shaft speed was also measured. Temperature conditions are monitored by thermocouple rakes in the flow and individual thermocouples in each tunnel wall panel. The wind tunnel and its associated instrumentation are fully automated and controlled using virtual instrument software created by EnFlo research staff using LabVIEW.

The 1:200 model installed in the wind tunnel is the simplest version of the DAPPLE site model, where all buildings have been reduced to simple blocks with flat roofs (see Figure 1-left). 
The boundary layer was generated using Irwin spires and surface roughness upwind of the model, obtaining a thickness of $1 \mathrm{~m}$ and a surface roughness $z_{0}=1.5 \mathrm{~mm}$ (equivalent to $0.3 \mathrm{~m}$ at full scale). The reference wind speed $\left(U_{r e f}\right)$ was set to $2.5 \mathrm{~m} \mathrm{~s}^{-1} ; U_{\text {ref }}$ was measured with the ultrasonic anemometer positioned just outside the simulated boundary layer. The model was oriented using a rotating turntable, and all the experiments were performed with a rotation of $51.35^{\circ}$ in model coordinates $\left(0^{\circ}\right.$ corresponds to a wind direction along the $x$ axis, see Figure 1left; model rotation is positive clockwise, hence the selected wind direction is approximately from south-west); the wind direction being chosen to correspond to conditions of the first field tracer release experiment (Arnold et al., 2004).

The total tracer flux, $\Phi$ through a reference plane $S$ is given by: , where $U_{n}$ is the mean flow speed normal to the surface, $C$ is the mean concentration (thus, $U_{n} C$ represents the mean tracer flux), and $\left\langle u_{n} c\right\rangle$ is the mean turbulent flux across the plane.

The experiments were performed using a Cambustion fast response (frequency $>350 \mathrm{~Hz}$ ) Flame Ionisation Detector (FFID) for concentration measurements, in conjunction with a twocomponent Dantec Laser Doppler Anemometer (LDA), for the velocity measurements (see Figure 1-right). This setup was capable of measuring the turbulent part of the flux along with the mean part at several locations within the urban model. The averaging time for the measurements was 1 minute as in previous measurements on the same model (Carpentieri et al., 2009; Carpentieri and Robins, 2010).

Five street canyon intersections (labelled as 'A', 'B', 'C', 'D' and ' $E$ ') were selected for tracer flux balance analysis; these are shown in Figure 2-left. The measurement sections (tagged with the initials of the street name and a number) and the various source positions (tagged as s1 to s7) used are also presented in this figure. Table 1 lists the characteristics of the experiments performed (tests T01-07). A further series of experiments was also carried out in order to analyse the sensitivity of the mass balance calculation versus the source location (tests SE1-4). The experiments were carried out using regular grids (spacing $=20 \mathrm{~mm}$; this is a compromise which allow a high spatial resolution while keeping the duration of the experiments to a minimum) across the selected sections within the canyons.

Vertical profiles were also measured at a number of locations along Gloucester Place in order to evaluate vertical fluxes within the street canopy.

\section{Vertical fluxes}

A series of vertical profiles of the turbulent flux of the tracer was measured within Gloucester Place (see Figure 2-right) - the LDA was aligned in the $x-z$ plane to measure the $U$ and $W$ components. Figures 3 shows some of the resulting profiles of the vertical turbulent tracer fluxes, along with the non-dimensional concentrations and concentration gradients (multiplied by an arbitrary proportionality factor $K=-1$ ). Concentrations have been non-dimensionalised using the expression $c^{*}=c U_{\text {ref }} H^{2} / Q$ (where $\mathrm{c}$ is the measured concentration, $\mathrm{H}$ is the boundary layer depth and $\mathrm{Q}$ is the tracer emission rate), while all velocities have been non-dimensionalised by using $\mathrm{U}_{\text {ref }}$ (for example $w^{*}=w / U_{\text {ref, }}$ where $w$ is the vertical velocity).

In general, the maximum of the vertical turbulent tracer flux $\left(w^{*} c^{*}\right)$ is located approximately at roof level, within the shear layer where the concentration gradient is also large.

Proportionality between concentration gradient and turbulent fluxes can be anticipated, as demonstrated by Dezső-Weidinger et al. (2003). Curves representing the concentration gradient multiplied by a factor $K=-1$ have been also reported in Figure 3, in order to facilitate the comparison with measured turbulent fluxes. A Gaussian fit to the measured concentrations has been used for calculating the gradients. As a matter of facts, commonly applied models usually compute turbulent fluxes from concentration gradients, even if the proportionality between these two parameters in urban settings has never been confirmed by experimental evidence.

The proportionality is apparent in the shown figures, and it is indeed true that the concentration gradients and the turbulent fluxes follow the same trend. However, the proportionality factor seems to be different for each measured vertical profile, depending on the 
local conditions. In particular, results from the 11 measured vertical profiles indicated a proportionality factor varying from -0.6 to -5.5 . This result indicates that the concentration gradient is indeed a main driver for the turbulent fluxes, but other factors might strongly affect them.

Analysing the figures in detail, it can be highlighted that greater vertical fluxes are found in locations (mainly within the canopy) where turbulence is also enhanced (for example in the wake of the tower above the bottom-left building of the intersection, see also Carpentieri et al., 2009), and where the velocity field is more complex (as in the wake of the buildings, particularly at the edges, see Figure 3-b). The local complexity of the velocity field is then another factor to be taken into account when computing turbulent fluxes.

It is worth noting that the shape of vertical profiles of turbulent tracer flux are comparable with those measured by Dezsö-Weidinger et al. (2003) using a different measurement technique. Unfortunately, a quantitative comparison is not possible, because only qualitative results were reported by Dezsö-Weidinger et al. (2003).

The results presented here highlight how the commonly used models to compute turbulent fluxes might strongly underestimate them when the local geometry leads to highly turbulent and complex velocity fields. The error will not be likely to affect the calculation of the total fluxes in places where advection is the main transport mechanism, but it may be significant for vertical exchanges.

\section{Mass flux balances}

Following the methodology applied by Carpentieri and Robins (2010), which relies on a linear interpolation for calculating the integral fluxes through a surface, a tracer flux balance was attempted for each of the five selected intersections (see Figure 2-left). The only difference with the technique used by Carpentieri and Robins (2010) is that, in the present case, the turbulent fluxes have also been considered in the balance. The results are presented in Figure 4.

The results are quite heterogeneous, with the distribution of the tracer strongly correlated with the local geometry. For example, intersections $\mathrm{C}$ and $\mathrm{D}$ have similar geometries and the distribution of pollutant between their streets is also rather similar. As found by Carpentieri and Robins (2010), however, local three-dimensional patterns strongly affect the distribution of pollutant: the path of the tracer from one street always intersects that of the tracer from another street. This means that a clear-cut separation of flow in the two canyons - as it is often assumed by even the most advanced operational mathematical models cannot be found; less advanced, though widely used models do not even take account of the exchange of pollutant between streets. For example, given the wind direction, one would expect the pollutant coming from Gloucester Place (from the south) to be carried into Marylebone Road (eastwards) at intersection A, whereas $7 \%$ of the tracer actually continues along Gloucester Place (northwards).

Turbulent fluxes are, in general, a small fraction of the overall tracer transfer. Percentages are usually lower than $3 \%$, with maxima of $6-8 \%$. The only exception is intersection $\mathrm{E}$, where the turbulent tracer flux through section MR2 accounts for $28 \%$ of the total. This is a confirmation of the very turbulent conditions occurring at this intersection and the strong entrainment flow into Upper Montague Street.

Since the measurements have not been performed over a closed volume, the calculated percentages are unbalanced. The differences (marked in the figures with a '?') may be due to exchange with the flow above the canopy, measurement errors and numerical error in the calculation (integration) procedures. In particular, higher uncertainty is expected for intersections $\mathrm{A}$ and $\mathrm{B}$ (Figure 4), where the complex geometry and the reduced number on measurement points in one of the inlet sections (especially SP5, due to the reduced size of the street) reduce the reliability of both the measurements and the integration process.

Estimating the total error is not easy, since we do not have higher resolution concentration and velocity fields to test the integration methodology. Repeatability tests have not been performed in this series of experiments, however previous studies on the same wind tunnel 
model (see, e.g., Carpentieri et al., 2009) suggest that the measurement error may estimated to be in the order of $10-15 \%$.

The most geometrically regular intersection is $\mathrm{C}$, surrounded by buildings with a similar height. At this intersection the difference between the incoming and outgoing fluxes is below $14 \%$. Due to this regularity, the smallest errors are expected here and thus the exchange with the flow above the canopy may be estimated to be of the order of $10 \%$, which is not unreasonable. Other intersections are less regular, with more complex flow leading, presumably, to an increased exchange. Unfortunately, errors are expected to increase as well and thus estimates of the exchange fluxes become less reliable.

Due to the high uncertainty in the data, a comparison with results from previous experiments (Carpentieri and Robins, 2010) on average mass fluxes is difficult. Only intersection A was analysed during that work and the order of magnitude of the tracer fluxes are similar.

\section{Source location sensitivity analysis}

Throughout our analysis, results have been considered independent of the emission source. While this is of course true for the source strength, since we are analysing nondimensional fluxes and concentrations, the location of the source with respect to the considered section could have an effect, especially if the initial dispersion of the tracer is far from uniform. An analysis of the sensitivity of the tracer flux balance calculation with the source location was performed, as reported in section 2. The tests were carried out at intersection A, both for sources in Marylebone Road (measurement sections MR5 and GP6) and in Gloucester Place (measurements sections GP5 and GP6). The results are summarised in Tables 2 and 3.

Despite first appearances, the two results are quite similar. In both cases the variation of the outgoing flux ratio is in the range of $\pm 15-20 \%$, despite the percentage variations in the fluxes calculated for each single section being of the order of $2-130 \%$. There is no recognisable trend and the variation in the calculation results seems to be affected by the complex flow around the source rather than the distance from the measurement sections. Overall the variations are of the same order of magnitude as the uncertainties.

\section{Conclusion and outlook}

Mean and turbulent tracer fluxes were measured within several street intersections in a wind tunnel model of a real urban area located in Central London. Simultaneous flow and tracer concentration measurement techniques were used. To our knowledge, this is the first attempt at directly measuring turbulent fluxes of tracer in urban areas.

The experiments were conducted in order to calculate tracer flux balances to help assess pollutant exchange between the streets of an intersection. Results are quite heterogeneous, despite the fact that only one wind direction was used and clearly demonstrate the strong influence of the intersection (and surrounding area) geometry. Generally, horizontal turbulent mass fluxes were found to be negligible with respect to the average mass flux driven by the mean flow advection mechanisms.

Measurements of vertical fluxes indicated an increase in turbulent exchange at roof level at the intersections, confirming the importance of this process in the exchange between canopy and external flow. A proportionality between turbulent fluxes and concentration gradients could be anticipated, however, the proportionality factor varied from -0.6 to -5.5 within the measured vertical profiles depending on their position. This suggests that current models might sensibly underestimate turbulent fluxes where the velocity field is more complex, and this is particularly significant for vertical fluxes, where advection plays only a minor role in transporting the pollutants.

Despite the apparently high spatial resolution of the measurement grid, uncertainties in the calculated fluxes were still large. However, a refinement of the technique based on the experience gained during this work will lead to more reliable results. In this way, reliable experimental data sets can be produced that will be vital in understanding and quantifying the 
exchange phenomena, a very important matter in urban air quality modelling, though one that is often neglected. One of the experimental difficulties lies in the variable 'roof level' of real urban areas and this imposes a rather complex control volume geometry, often with a number of small sub-faces. A very fine resolution is needed to manage these situations.

\section{References}

Arnold S.J., ApSimon H., Barlow J., Belcher S., Bell M., Boddy J.W., Britter R., Cheng H., Clark R., Colvile R.N., Dimitroulopoulou S., Dobre A., Greally B., Kaur S., Knights A., Lawton T., Makepeace A., Martin D., Neophytou M., Neville S., Nieuwenhuijsen M., Nickless G., Price C., Robins A., Shallcross D., Simmonds P., Smalley R.J., Tate J., Tomlin A.S., Wang H., Walsh P., 2004. Introduction to the DAPPLE air pollution project. Science of the Total Environment 332, 139-153.

Barlow J.F., Belcher S.E., 2002. A wind tunnel model for quantifying fluxes in the urban boundary layer. Boundary-Layer Meteorology 104, 131-150.

Barlow J.F., Harman I.N., Belcher S.E., 2004. Scalar fluxes from urban street canyons.

Part I: Laboratory simulation. Boundary-Layer Meteorology 113, 369-385.

Britter R.E., Hanna S.R., 2003. Flow and dispersion in urban areas. Annual Review of Fluid Mechanics 35, 469-496.

Carpentieri M., Robins A.G., Baldi S., 2009. Three-dimensional mapping of air flow at an urban canyon intersection. Boundary-Layer Meteorology 133, 277-296.

Carpentieri M., Robins A.G., 2010. Tracer flux balance at an urban canyon intersection. Boundary-Layer Meteorology 135, 229-242.

Caton F., Britter R.E., Dalziel S., 2003. Dispersion mechanisms in a street canyon. Atmospheric Environment 37, 693-702.

Dezső-Weidinger G., Stitou A., Van Beeck J., Riethmuller M.L., 2003. Measurement of the turbulent mass flux with PTV in a street canyon. Journal of Wind Engineering and Industrial Aerodynamics 91, 1117-1131.

Fackrell, J.E. and Robins, A.G., 1982. Concentration fluctuations and fluxes in plumes. J. Fluid Mech., 117, 1-26.

Hoydysh W.G., Dabberdt W.F., 1994. Concentration fields at urban intersections: fluid modeling studies. Atmospheric Environment 28, 1849-1860.

Lemoine F., Wolff M.., Lebouché M., 1997. Experimental investigation of mass transfer in a grid-generated turbulent flow using combined optical methods. International Journal of Heat and Mass Transfer 40, 3255-3266.

Narita K., 2007. Experimental study of the transfer velocity for urban surfaces with a water evaporation method. Boundary-Layer Meteorology 122, 293-320.

Pascheke F., Barlow J.F., Robins A., 2008. Wind-tunnel modelling of dispersion from a scalar area source in urban-like roughness. Boundary-Layer Meteorology 126, 103-124.

Soulhac L., 2000. Modélisation de la dispérsion atmosphérique a l'interieur de la canopée urbaine. PhD thesis, Ecole Centrale de Lyon, France.

Wood C.R., Arnold S.J., Balogun A.A., Barlow J.F., Belcher S., Britter R.E., Cheng H., Dobre A., Lingard J.J.N., Martin D., Neophytou M.K., Petersson F.K., Robins A.G., Shallcross D.E., Smalley R.J., Tate J.E., Tomlin A.S., White I.R., 2009. Dispersion experiments in Central London: the 2007 DAPPLE project. Bulletin of the American Meteorological Society 90, 955969.

Zhu J.Y., So R.M.C., Otugen M.V., 1988. Turbulent mass flux measurements using a laser/hot-wire technique. International Journal of Heat and Mass Transfer 31, 819-829. 


\section{Figures}
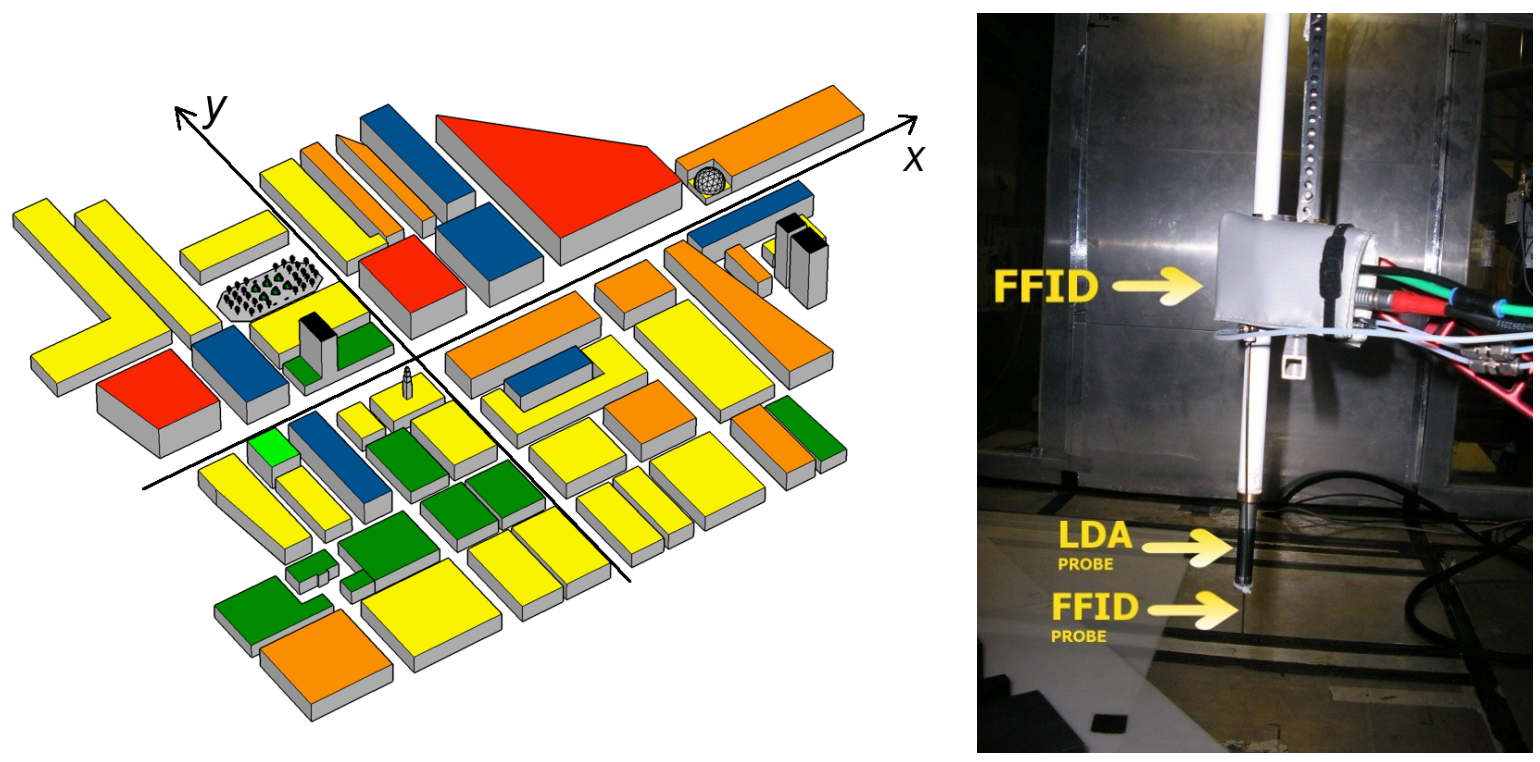

Figure 1 - Left: three-dimensional representation of the 1:200 DAPPLE model; the grey shades correspond to different building height ranges. Right: instrumentation used for the concentration and velocity measurements
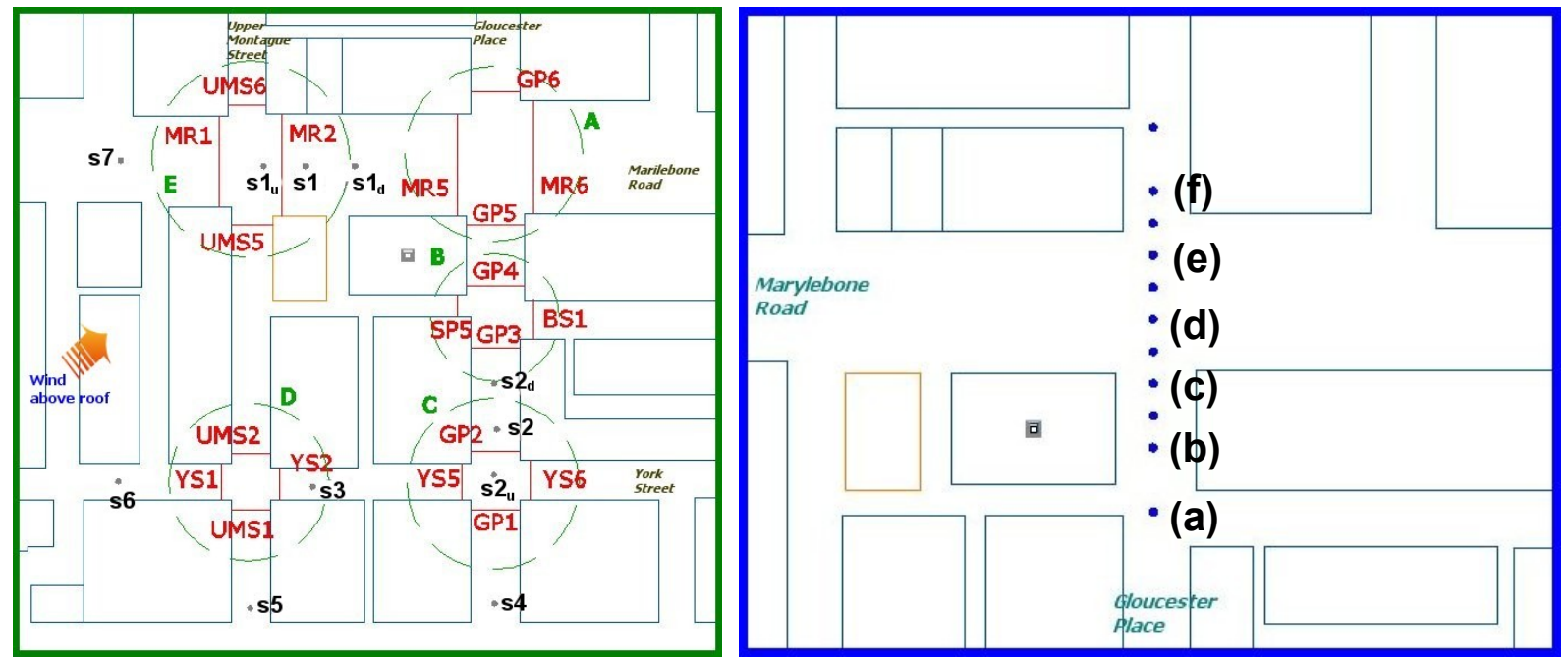

Figure 2 - Intersections analysed, measurement sections and source locations for the experiments (left); map of the locations of vertical profile measurements (right; the letters correspond to the vertical profiles presented in Figure 3) 
(a)

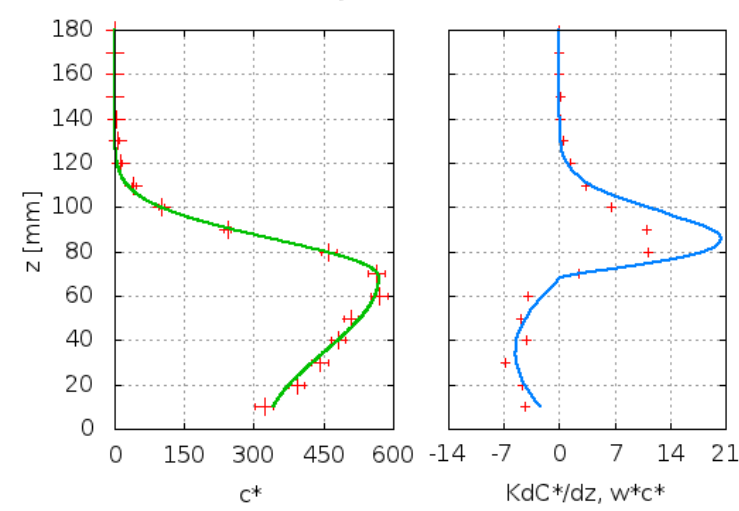

(c)
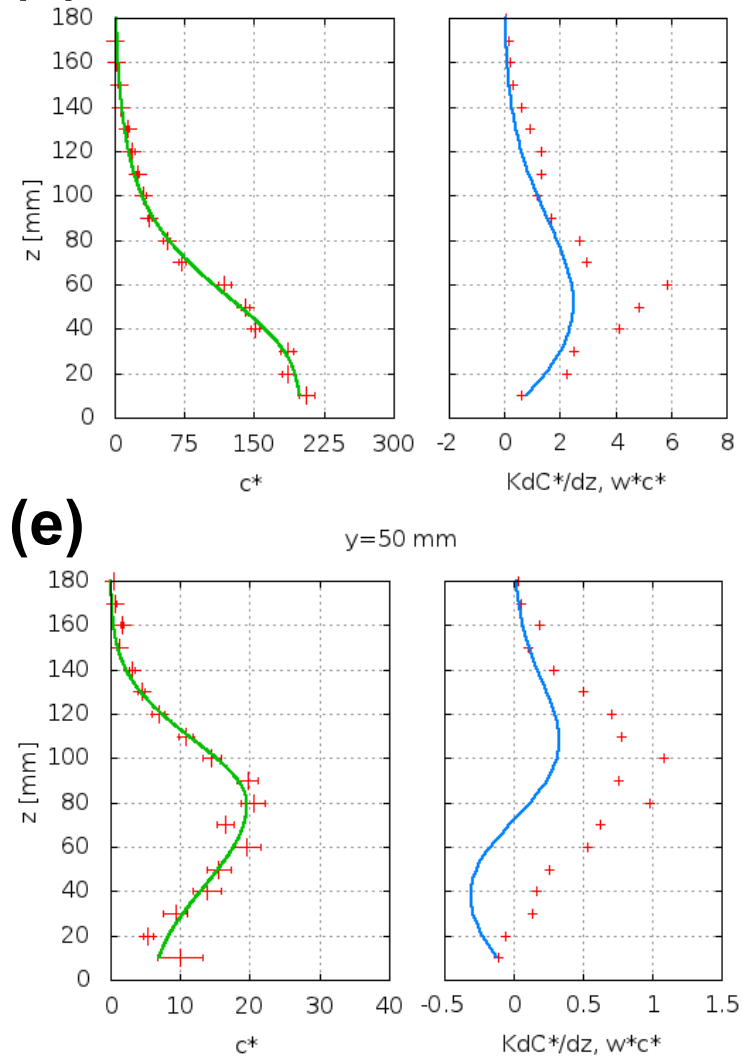

(b)

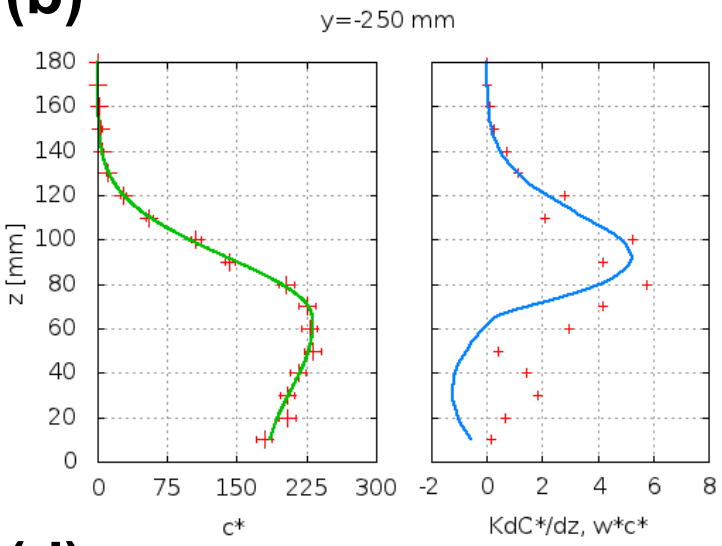

(d)

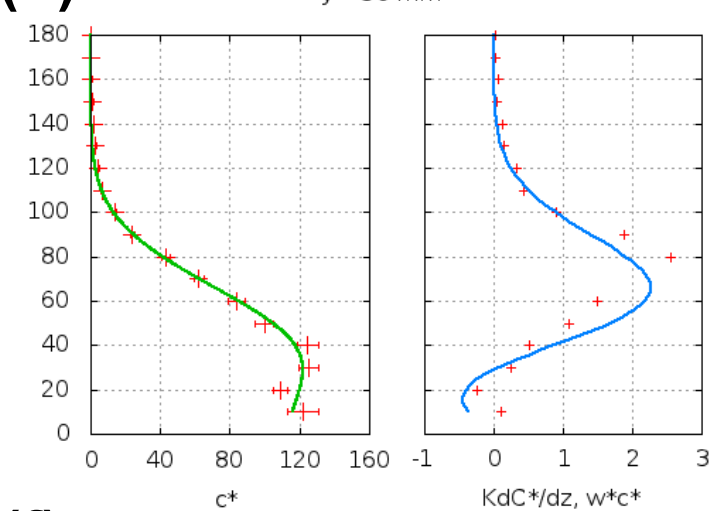

(f)

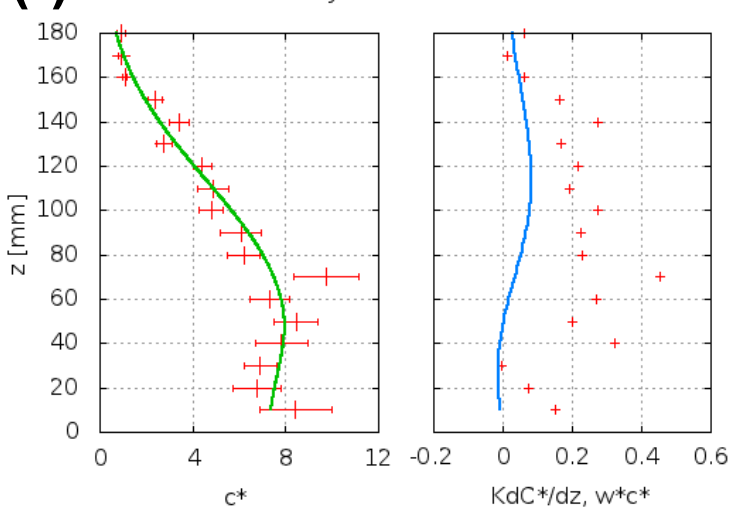

Figure 3 - Selected vertical profiles of tracer concentration $\left(c^{*}\right)$, and tracer turbulent flux $\left(w^{*} c^{*}\right)$ along Gloucester Place; in the $c^{*}$ graphs, the line represents a Gaussian fit to the measured concentrations, while the line in the $w^{*} c^{*}$ graphs is the concentration gradient calculated from the Gaussian fit and multiplied by a factor $K=-1$. 

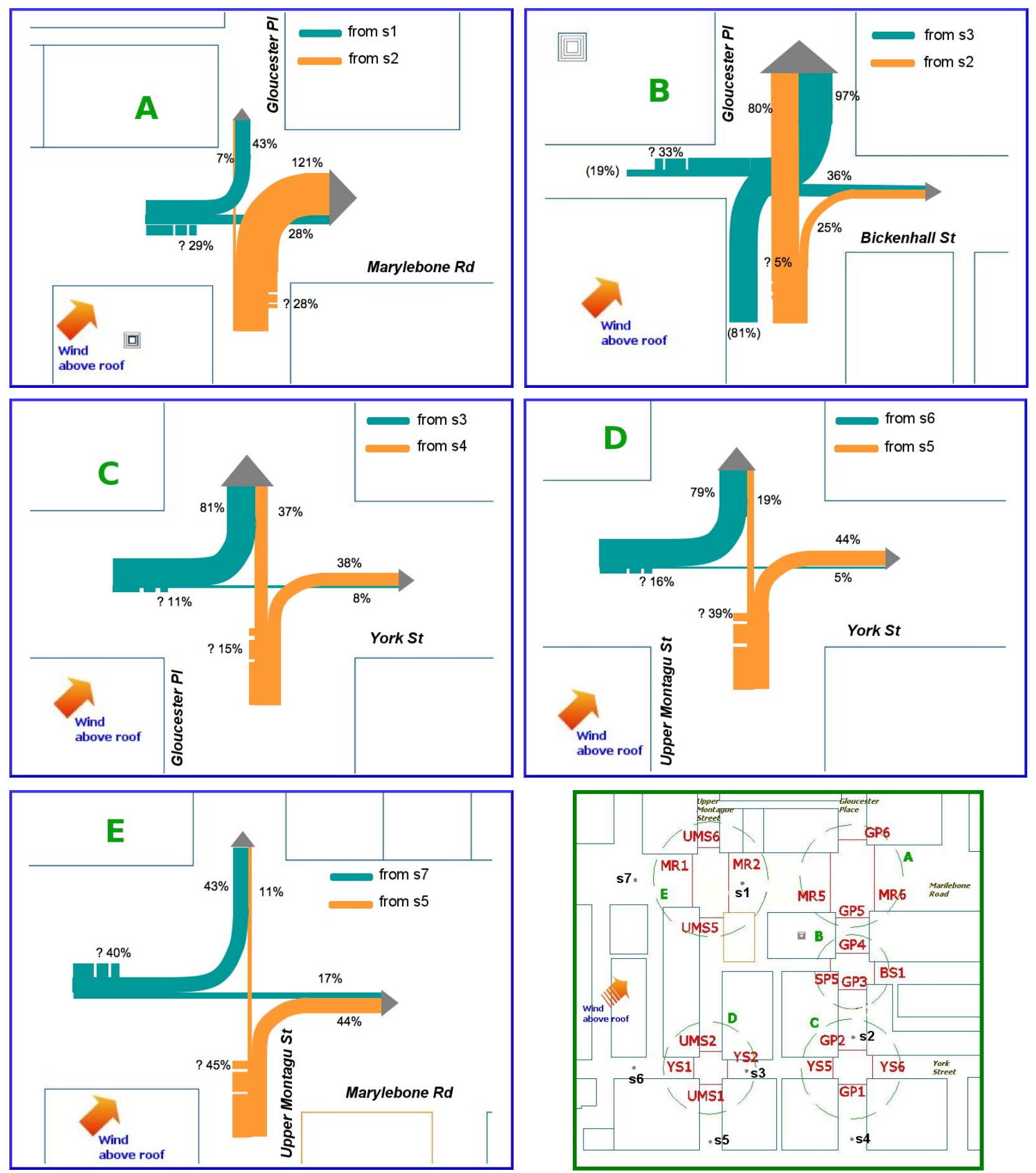

Figure 4 - Mass flux balance at the five studied intersections (located as represented in the map at bottom-right). Different gray shades represent fluxes from different emission sources (s1 to s7). Notation for the intersection and sections labelling is explained in Table 1. 


\section{Tables}

Table 1 - List of test configurations

Table 2 - Results of the sensitivity analysis for the source in Marylebone Road; mean and turbulent tracer fluxes are in non-dimensional form

Table 3 - Results of the sensitivity analysis for the source in Gloucester Place; mean and turbulent tracer fluxes are in non-dimensional form

Table 1:

\begin{tabular}{|c|c|c|c|}
\hline Test & Intersections & Sections & Source \\
\hline T01 & A & MR5-6,GP6 & s1 \\
\hline T02 & A,B & GP3-4-5-6,MR6,BS1 & s2 \\
\hline T03 & B,C & GP2-3-4,YS5-6,SP5,BS1 & s3 \\
\hline T04 & C & GP1-2,YS6 & s4 \\
\hline T05 & D,E & UMS1-2-5-6,YS2,MR2 & $\mathrm{s} 5$ \\
\hline T06 & D & UMS2,YS1-2 & $\mathrm{s} 6$ \\
\hline T07 & E & UMS6,MR1-2 & $\mathrm{s} 7$ \\
\hline SE1 & A & MR5,GP6 & $\mathrm{s} 1_{\mathrm{d}}$ \\
\hline SE2 & A & GP5-6 & $\mathrm{s} 1_{\mathrm{u}}$ \\
\hline SE3 & A & GP5-6 & $\mathrm{s} 2_{\mathrm{d}}$ \\
\hline SE4 & A & $\mathrm{s} 2_{\mathrm{u}}$ \\
\hline
\end{tabular}

Table 2:

\begin{tabular}{|c|c|c|c|c|c|}
\hline Source-section & Mean flux & $\begin{array}{c}\text { Turbulent } \\
\text { flux }\end{array}$ & $\begin{array}{c}\text { Turbulent/mean } \\
\text { flux ratio }\end{array}$ & In flux & Out flux \\
\hline $\mathrm{s} 1_{\mathrm{d}}-\mathrm{MR5}$ & 0.29 & -0.0086 & $-2.91 \%$ & $100 \%$ & - \\
$\mathrm{s} 1_{\mathrm{d}}-\mathrm{GP} 6$ & 0.17 & -0.010 & $-5.81 \%$ & - & $57 \%$ \\
\hline $\mathrm{s} 1-\mathrm{MR} 5$ & 0.18 & -0.0015 & $-0.86 \%$ & $100 \%$ & - \\
$\mathrm{s} 1-\mathrm{GP} 6$ & 0.080 & -0.0046 & $-5.78 \%$ & - & $43 \%$ \\
\hline $\mathrm{s} 1_{\mathrm{u}}-\mathrm{MR} 5$ & 0.15 & -0.0011 & $-0.73 \%$ & $100 \%$ & - \\
$\mathrm{S} 1_{\mathrm{u}}-\mathrm{GP} 6$ & 0.098 & -0.0050 & $-5.16 \%$ & - & $63 \%$ \\
\hline
\end{tabular}

Table 3:

\begin{tabular}{|c|c|c|c|c|c|}
\hline Source-section & Mean flux & $\begin{array}{c}\text { Turbulent } \\
\text { flux }\end{array}$ & $\begin{array}{c}\text { Turbulent/mean } \\
\text { flux ratio }\end{array}$ & In flux & Out flux \\
\hline $\mathrm{s} 2{ }_{\mathrm{d}}-\mathrm{GP} 5$ & 0.63 & -0.013 & $-1.98 \%$ & $100 \%$ & - \\
$\mathrm{s} 2 \mathrm{~d}-\mathrm{GP} 6$ & 0.051 & -0.0017 & $-5.80 \%$ & - & $8 \%$ \\
\hline $\mathrm{s} 2-\mathrm{GP} 5$ & 0.45 & -0.0026 & $-0.56 \%$ & $100 \%$ & - \\
$\mathrm{s} 2-\mathrm{GP} 6$ & 0.036 & -0.0024 & $-6.69 \%$ & - & $7 \%$ \\
\hline $\mathrm{s} 2{ }_{\mathrm{u}}-\mathrm{GP} 5$ & 0.33 & -0.0046 & $-1.39 \%$ & $100 \%$ & - \\
$\mathrm{s} 2{ }_{\mathrm{u}}-\mathrm{GP} 6$ & 0.033 & -0.0017 & $-5.20 \%$ & - & $10 \%$ \\
\hline
\end{tabular}

